\title{
BMJ Open Study protocol for a randomised controlled feasibility trial of a virtual intervention (STRIDE) for symptom management, distress and adherence to adjuvant endocrine therapy after breast cancer
}

\author{
Jamie M Jacobs (DD , ${ }^{1,2}$ Chelsea S Rapoport, ${ }^{1}$ Arielle Horenstein, ${ }^{1,3}$ Madison Clay, ${ }^{1}$ \\ Emily A Walsh,, ${ }^{1,4}$ Jeffrey Peppercorn,, ${ }^{5,6}$ Jennifer S Temel, ${ }^{5,6}$ Joseph A Greer $^{1,2}$
}

To cite: Jacobs JM,

Rapoport CS, Horenstein A, et al. Study protocol for a randomised controlled feasibility trial of a virtual intervention (STRIDE) for symptom management, distress and adherence to adjuvant endocrine therapy after breast cancer. BMJ Open 2021;11:e041626. doi:10.1136/ bmjopen-2020-041626

- Prepublication history and additional material for this paper are available online. To view these files, please visit the journal online (http://dx.doi. org/10.1136/bmjopen-2020041626).

Received 12 June 2020 Revised 01 December 2020 Accepted 09 December 2020

Check for updates

(C) Author(s) (or their employer(s)) 2021. Re-use permitted under CC BY-NC. No commercial re-use. See rights and permissions. Published by BMJ.

For numbered affiliations see end of article.

Correspondence to Dr Jamie M Jacobs; jjacobs@mgh.harvard.edu

\section{ABSTRACT}

Introduction Patient adherence to adjuvant endocrine therapy (AET) after a diagnosis of hormone-sensitive breast cancer is poor. Previous interventions have failed to produce changes in adherence, address patient preferences or include theoretically informed and evidence-based components. Therefore, we iteratively developed a patient-centred, evidence-based, smallgroup, videoconference intervention to improve adherence and symptom management as well as reduce distress for patients taking AET after breast cancer (SymptomTargeted Randomised Intervention for Distress and Adherence to Adjuvant Endocrine Therapy, STRIDE). Methods and analysis The current study is a nonblinded, randomised, controlled, feasibility trial of STRIDE compared with a medication monitoring control group. The primary objective is to examine the feasibility and acceptability of STRIDE, while secondary objectives are to assess changes in objective and subjective adherence, symptom distress and satisfaction with AET. Patients will be recruited from the Massachusetts General Hospital Cancer Center in Boston, Massachusetts. The total number of patients accrued will be 75 , with $\geq 60$ patients completing the study. All patients will store their AET in an electronic pill bottle for objective adherence monitoring. Patients randomly assigned to the STRIDE intervention will receive 6 weekly 1 -hour sessions, in small groups of two, delivered via videoconferencing by a trained mental health professional. Patients assigned to the control group will store their medication in the electronic pill bottle and receive follow-up oncology care as usual. All participants will complete self-report psychosocial measures at baseline, 12 weeks and 24 weeks postbaseline.

Ethics and dissemination The study is funded by the National Cancer Institute of the National Institutes of Health and is approved by the Dana-Farber/Harvard Cancer Center Institutional Review Board (Protocol \#18-603, V.1.2, first approval date 1 February 2019). The study will be reported in accordance with the Consolidated Standards of Reporting Trials statement for non-pharmacological trials. Results will be published in peer-reviewed
Strengths and limitations of this study

- This study employs a patient-centred, evidencebased, virtual videoconference intervention to improve adherence, enhance symptom management, and reduce distress for patients taking adjuvant endocrine therapy after breast cancer.

- Given the lack of efficacious interventions to date, this study design and intervention were informed by in-depth qualitative analysis and iterative intervention development to maximise patient-centredness, feasibility, acceptability and efficacy.

- To maximise scalability and dissemination, patients participate virtually for all study procedures including consent, intervention and assessments; therefore, no hospital or in-person visits are necessary.

- The homogeneous sample with respect to sociodemographic diversity will limit generalisability of the findings.

academic journals, presented at scientific meetings and disseminated to patient organisations and media outlets. Trial registration number NCT03837496; Pre-results.

\section{INTRODUCTION}

Up to $75 \%$ of female patients diagnosed with early-stage breast cancer will require adjuvant endocrine therapy (AET) to prevent the likelihood of recurrence and improve survival. ${ }^{1}$ AET (eg, tamoxifen or an aromatase inhibitor) reduces recurrence and mortality by approximately $30 \%-50 \%$, for hormone receptor-positive breast cancer. ${ }^{2}{ }^{3}$ Despite the substantial, indisputable benefit, half of women are non-adherent (ie, do not take the medication as prescribed) within 5 years of initiating AET. ${ }^{4}$ In addition to greater risk of 
breast cancer recurrence ${ }^{56}$ and breast cancer mortality, ${ }^{7}$ non-adherence to AET is associated with higher rates of physician visits, hospitalisations ${ }^{8}$ and poorer patientprovider relationships. ${ }^{9}$

Several factors are known to contribute to suboptimal adherence for patients taking AET, ${ }^{510}$ including substantial side effects (eg, joint pain, hot flashes, sleep difficulties), ${ }^{11-13}$ psychological distress during survivorship, ${ }^{11}$ low perceived need for AET, ${ }^{14}$ low self-efficacy for taking medication, ${ }^{15}$ negative beliefs about AET and related side effects ${ }^{16}$ and low social support. ${ }^{17}$ While several of these contributors are modifiable, patients receive virtually no formal support to promote medication-taking after breast cancer. Only four randomised controlled trials (RCTs) to date have investigated psychosocial interventions to promote AET adherence, ${ }^{18}$ and they have failed to address the adherence challenges that patients face. Previous studies neglected to include theoretically driven and empirically based intervention components to target behavioural drivers of adherence, were not developed using guiding principles of behavioural intervention development, ${ }^{19}{ }^{20}$ have been methodologically flawed, and were not informed by patient preferences.

To address the significant need to optimise AET adherence, improve self-management of side effects and reduce distress for patients taking AET, we iteratively developed a patient-centred, small-group, evidencebased, videoconference intervention: SymptomTargeted Randomised Intervention for Distress and Adherence to Adjuvant Endocrine Therapy (STRIDE). Intervention development was informed by a comprehensive systematic literature review, ${ }^{18}$ a qualitative in-depth analysis of patient experiences and preferences, ${ }^{21}$ prior efficacious treatments ${ }^{22}{ }^{23}$ and expert feedback from oncology clinicians and behavioural scientists. We then modified intervention content and duration, logistics, and study procedures using quantitative and qualitative feedback from exit interviews with five patients who participated in a run-in phase of the intervention. Patients were enthusiastic about the STRIDE intervention, described it as beneficial, preferred the group setting and noted that the virtual delivery was a necessary convenience.

The current report outlines the details of the RCT that is currently underway comparing the finalised STRIDE intervention to a medication monitoring control condition. The primary objective is to establish the feasibility and acceptability of the STRIDE intervention compared with the medication monitoring control group. The secondary aims are to assess the effects of the intervention on objective and self-reported AET adherence, symptom distress and satisfaction with AET. Finally, we will explore group differences on additional self-reported psychosocial constructs and examine potential mediators and moderators of any treatment outcomes. This pilot study will ultimately inform a future full-scale trial which will examine intervention efficacy.
Screening, approach, and consent

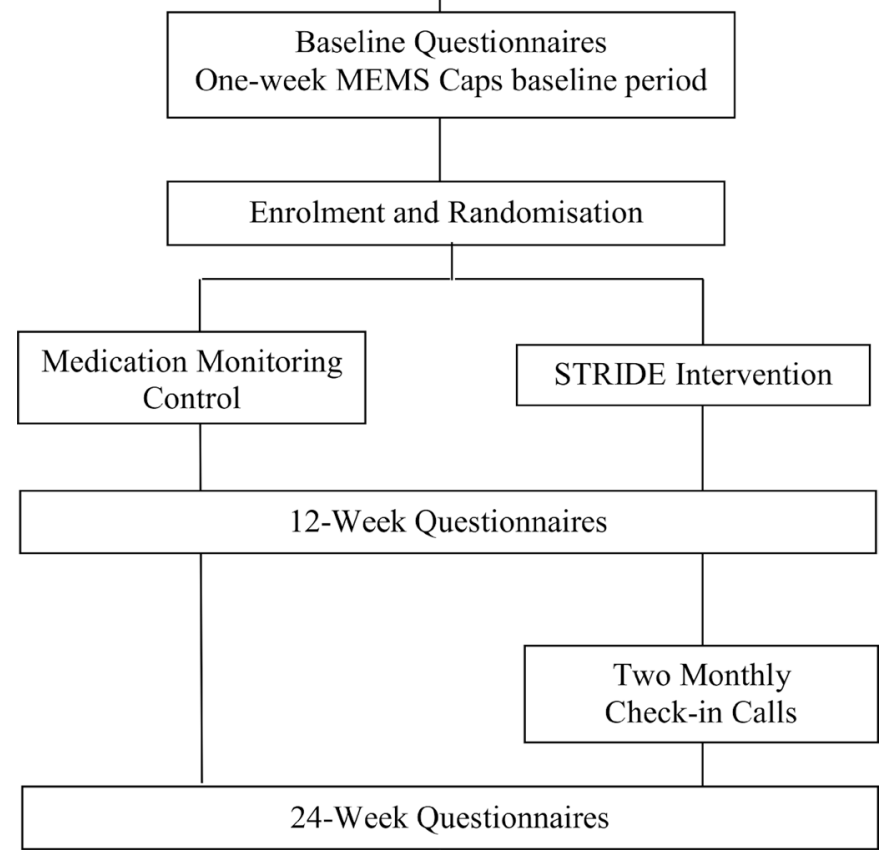

Figure 1 CONSORT flow diagram. CONSORT, Consolidated Standards of Reporting Trials; MEMS, Medication Event Monitoring System; STRIDE, Symptom-Targeted Randomised Intervention for Distress and Adherence to Adjuvant Endocrine Therapy.

\section{METHODS AND ANALYSIS \\ Study design}

This is a single-site, randomised, controlled, pilot feasibility study comparing a videoconference intervention (STRIDE) to standard care plus medication monitoring in 75 patients taking AET after breast cancer. Recruitment will occur at the Massachusetts General Hospital (MGH) Boston and three MGH community affiliates: MGH Newton-Wellesley, MGH North Shore and MGH Waltham. The Consolidated Standards of Reporting Trials flow diagram is illustrated in figure 1 . The study is approved by the Dana Farber/Harvard Cancer Center's (DF/HCC) Institutional Review Board.

\section{Participant selection}

To be eligible, patients must be female, be age 21 or older, have a diagnosis of early-stage (stage 0 -IIIb), have hormone receptor-positive breast cancer, have completed primary treatment (ie, chemotherapy, surgery and/ or radiation) for breast cancer, be within 1 week to 36 months of starting AET, be able to read and respond in English, and have an Eastern Cooperative Oncology Group performance status $\leq 2$ (see box 1). Patients will complete a 3-item distress screening using an adapted National Comprehensive Cancer Network Distress Thermometer (range $=0-10) .{ }^{24}$ Those who report distress $\geq 4$ on any of the three items will be eligible to participate (ie, distress related to having to take AET, distress related 


\section{Box 1 Eligibility criteria}

\section{Inclusion criteria \\ 1. Female.}

2. Age 21 or older.

3. Diagnosis of early-stage (stage $0-1 \mathrm{llb}$ ), hormone receptor+breast cancer.

4. Within 1 week to 36 months of starting adjuvant endocrine therapy. 5. Ability to read and respond in English.

6. Eastern Cooperative Oncology Group performance status $\leq 2$.

7. Currently taking adjuvant endocrine therapy (ie, if took recent break, has taken within the past 2 weeks).

8. Completed primary treatment (ie, chemotherapy, surgery and/or radiation) for early-stage breast cancer.

9. Indicates a score $\geq 4$ on one of the three National Comprehensive Cancer Network adapted distress thermometer study screening questions.

\section{Exclusion criteria}

1. Uncontrolled psychosis, active suicidal ideation or psychiatric hospitalisation within the past year.

2. Cognitive impairment that prohibits participation in the study.

3. Enrolment in a different clinical trial for breast cancer.

4. Current participation in formal group psychotherapy or other psychosocial intervention trial.

to symptoms/side effects and/or distress related to AET adherence). To ensure engagement and compliance with study procedures, patients with cognitive impairment, uncontrolled psychosis, active suicidal ideation or a psychiatric hospitalisation within the past year will not be eligible. In addition, exclusion criteria include enrolment in a clinical trial for breast cancer (due to additional medication monitoring), participation in another psychosocial intervention study or current engagement in formal group psychotherapy.

Five patients participated in a trial run-in phase, and 75 will participate in the RCT. We estimate that the enrolment rate will be approximately $60 \%$. If 134 patients are approached and 80 (total) are enrolled, the lower limit for an exact, one-sided 95\% CI for the estimated enrolment rate will be $53 \%$. Furthermore, we anticipate that the retention and attendance rates will both be at least $80 \%$, and with 80 enrolled participants, the lower limit for an exact, one-sided $95 \%$ CI for the retention and attendance rates will be $71 \%$. Thus, based on our estimates of the feasibility parameters, the study will demonstrate feasibility of the STRIDE intervention with a sample size of 75 in the RCT and five in the run-in.

\section{Study procedures}

\section{Recruitment}

Trained study staff will review the electronic health record (EHR) to identify potentially eligible patients with upcoming appointments at the MGH Center for Breast Cancer or one of the MGH community affiliates. For those identified patients, study staff will then request permission from the respective oncology clinicians to approach the patients about study participation. After receiving approval from the oncology clinicians, study staff will approach patients during their outpatient appointment to explain study procedures and gauge their interest in participating. Given the heavy volume of patients in the breast clinic on any given day, study staff can also contact patients by telephone if they are not able to approach them in person. Patients can also self-refer through advertisements on the online MGH study recruitment site or via posted flyers in the clinic. Finally, the oncology clinician or nurse practitioner can also directly refer interested patients.

\section{Enrolment and randomisation}

Eligible patients approached in person will complete written informed consent with trained study staff, and those approached by telephone will complete electronic informed consent with trained study staff. Enrolled patients will complete a 1-week baseline period during which they will use the Medication Event Monitoring System (MEMS Caps), ${ }^{25}$ to electronically monitor medication-taking, as well as complete baseline self-report study questionnaires. Following 1 week of medication monitoring, study staff will randomly allocate patients 1:1 to either the STRIDE intervention or the medication monitoring control group via a computer-generated randomisation scheme created by the study biostatistician; group allocation will be concealed for each patient until randomisation occurs. Randomisation will be stratified according to level of distress, determined by baseline scores on the Hospital Anxiety and Depression Scale (HADS),${ }^{26}$ (high distress $\geq 8$; low distress $<8$ ).

\section{Assessments}

Patients will complete the same self-report measures at 12-week and 24-week follow-ups. Assessments will be conducted using mailed paper questionnaires or electronically via Research Electronic Data Capture (REDCap), a Health Insurance Portability and Accountability Act compliant, web-based survey tool. ${ }^{27}$ Given the virtual nature of the assessments and intervention sessions, patients will not need to travel to the hospital for any study-related visits and will receive US\$20 per assessment for their time and effort.

\section{STRIDE Intervention}

STRIDE is a brief, small group-based, videoconference intervention with 6weekly 1-hour sessions and two follow-up 15-30 min telephone check-ins occurring approximately one and 2 months after the final intervention session. Groups will include two patients, matched according to schedules, and will take place via $\mathrm{MGH}$-approved videoconference software, Zoom Video Communications, Inc (Zoom). The STRIDE intervention incorporates aspects of Cognitive-Behavioural Therapy for Adherence and Depression ${ }^{28}$ and CognitiveBehavioural Stress Management for Breast Cancer, ${ }^{22}$ such as cognitive restructuring, behavioural activation, relaxation training and skills-based coping strategies to address adherence and mood symptoms. The intervention also 
includes psychoeducation about the benefits and risks of AET, an assessment of barriers to adherence, and problem solving of individual barriers. Three of the six sessions focus entirely on addressing AET-related side effects and breast cancer treatment-related symptoms, such as hot flashes, pain and fatigue, through specific behavioural coping strategies, evidence-based symptom management and problem solving. As part of the intervention, participants will be asked to practice coping skills and relaxation training, using audio recordings, in between sessions.

\section{Patient and public involvement}

Patients were involved in an initial qualitative phase of this study, which informed the overall study design, intervention and outcomes measures. Briefly, 30 patients enrolled and engaged in semistructured interviews with trained study staff to understand experiences with and perceptions of AET, barriers and facilitators to adherence, emotional and symptom-related distress. ${ }^{21}$ Interviews also solicited feedback from patients about their preferences for a psychosocial intervention, such as whether they preferred videoconference vs in person, individual versus group and session length, frequency and duration. Recurrent themes from these interviews informed intervention content and procedures. In addition, five patients participated in a run-in trial of the intervention and assessment measures and quantitatively rated the acceptability, enjoyableness and feasibility of the intervention while also completing semistructured interviews to provide feedback about the intervention, assessments and study procedures. The intervention and procedures were modified and refined based on feedback from these exit interviews. For example, an additional session was added to the intervention for a total of six sessions in the final intervention, and an outcome measure was added based on feedback about acceptability of the length of the assessment battery.

\section{Intervention delivery}

Trained licensed psychologists, licensed social workers and psychology doctoral students will administer the intervention and participate in weekly clinical group supervision with the principal investigator (PI). To monitor treatment fidelity, at least $10 \%$ of sessions will be randomly selected, stratified by study therapist and reviewed by an independent assessor for percentage of key intervention topics covered, with a goal of $90 \%$ of possible topics covered per session. ${ }^{29}$ Feedback will be discussed with the study therapists to maintain and enhance adherence to the intervention manual.

\section{Medication monitoring control}

Participants in the control condition will monitor medication-taking with MEMS Caps and otherwise receive follow-up oncology care as usual. As medication monitoring can increase adherence in and of itself, ${ }^{30}$ both groups will self-monitor medication and only participants randomly assigned to STRIDE will receive the intervention.

\section{Outcomes}

Table 1 lists the self-report questionnaires and the time points at which they are administered.

\section{Demographic and clinical characteristics}

Participants will self-report their age, gender, race, ethnicity, marital status, education level and relationship status. The following information will be collected from the EHR: MGH clinic site, breast cancer stage, node status, HER2/neu status, treatment type (eg, surgery, chemotherapy, radiation), time since treatment completion, AET medication type, date of AET initiation, menopausal status, ovarian suppression (yes/no), oophorectomy (yes/no) and number of concomitant medications at study enrolment.

\section{Primary outcomes}

Feasibility and acceptability. Feasibility will be measured by the rate of enrolment $(>50 \%)$, participant retention $(>70 \%)$ and intervention attendance $(\geq 70 \%$ of patients attending at least four of six sessions). Acceptability will be measured by intervention satisfaction, with $>75 \%$ of patients reporting average satisfaction greater than the midpoint of the Client Satisfaction Questionnaire at the 12 week assessment. ${ }^{31}$

\section{Secondary outcomes}

Adherence to AET. Objective adherence rates will be recorded using the MEMS Caps, ${ }^{25}$ which are widely used in adherence monitoring, including for patients with breast cancer. ${ }^{32-34}$ Patients store their medication in the MEMS bottle, which records the date and time of any openings as a proxy for medication taking. The supplemental medication diary will be used alongside the MEMS Caps to document any instance in which a patient takes the medication without opening the MEMS Cap. We will also make note of any physician-prescribed medication break during the patients' study participation. We will further assess self-reported adherence using the five-item Medication Adherence Report Scale (MARS-5). ${ }^{35}$ The MARS-5 measures adherence to treatment with five questions concerning forgetting, changing doses, stopping, skipping and using less than what is prescribed.

Symptom distress. We will assess symptom distress using the Breast Cancer Prevention Trial Symptom Scale, ${ }^{36}$ a symptom checklist for which patients document how much they were bothered by several physical and psychological symptoms associated with AET use, such as hot flashes, weight gain, night sweats and joint pain.

Satisfaction with AET. We will assess satisfaction with AET using the Cancer Therapy Satisfaction Questionnaire (CTSQ) ${ }^{37}$ The CTSQ is a self-report measure that evaluates patients' beliefs about specific aspects of the medication, such as expectations of the effectiveness of AET therapy, feelings about side effects and therapy adherence. 
Table 1 Study instruments and time points

\begin{tabular}{|c|c|c|c|c|}
\hline Instrument/measure & Screening & $\begin{array}{l}\text { Baseline } \\
\text { ( } 4 \text { weeks window } \\
\text { from consent) }\end{array}$ & $\begin{array}{l}12 \text { weeks } \\
\text { postbaseline } \\
\text { ( } \pm 2 \text { weeks } \\
\text { window) }\end{array}$ & $\begin{array}{l}24 \text { weeks } \\
\text { postbaseline } \\
( \pm 2 \text { weeks } \\
\text { window) }^{\star}\end{array}$ \\
\hline Adapted NCCN Distress Thermometer & $\mathrm{X}$ & & & \\
\hline Medication Event Monitoring System (MEMS Caps) & & \multicolumn{3}{|c|}{ To be used throughout the 24-week study period } \\
\hline Medication Adherence Report Scale-5 & & $\mathrm{x}$ & $\mathrm{x}$ & $x$ \\
\hline Breast Cancer Prevention Trial Symptom Checklist & & $\mathrm{X}$ & $\mathrm{X}$ & $\mathrm{X}$ \\
\hline Cancer Therapy Satisfaction Questionnaire & & $\mathrm{x}$ & $\mathrm{x}$ & $\mathrm{X}$ \\
\hline Hospital Anxiety and Depression Scale & & $\mathrm{X}$ & $\mathrm{X}$ & $\mathrm{X}$ \\
\hline Functional Assessment of Cancer Therapy & & $x$ & $\mathrm{x}$ & $x$ \\
\hline Multidimensional Scale of Perceived Social Support & & $\mathrm{X}$ & $\mathrm{X}$ & $\mathrm{X}$ \\
\hline Self-Efficacy in Appropriate Medication Use Scale & & $\mathrm{X}$ & $\mathrm{X}$ & $\mathrm{X}$ \\
\hline $\begin{array}{l}\text { Self-Efficacy For Managing Symptoms and Taking AET } \\
\text { Questionnaire (Self-Efficacy For Symptoms) }\end{array}$ & & $x$ & $x$ & $x$ \\
\hline $\begin{array}{l}\text { Patient-Reported Outcomes Measurement Information } \\
\text { System - Cognitive Function - Short Form } 4 a\end{array}$ & & $\mathrm{x}$ & $\mathrm{X}$ & $\mathrm{x}$ \\
\hline Client Satisfaction Questionnaire (CSQ)* & & & $\mathrm{X}$ & \\
\hline Supplemental Medication Diary & $\begin{array}{l}\text { To serve as } \\
\text { vacation) }\end{array}$ & optional as-needed s & supplement to 1 & S Caps (eg, on \\
\hline
\end{tabular}

${ }^{*} \mathrm{CSQ}$ will be administered to intervention participants only.

NCCN, National Comprehensive Cancer Network.

\section{Exploratory outcomes}

The following measures will be administered to explore group differences on several psychosocial constructs, as well as possible mediators or moderators of intervention effects: quality of life (QOL) measured by the Functional Assessment of Cancer Therapy-Breast Cancer, ${ }^{38}$ medication-taking self-efficacy measured by the SelfEfficacy for Appropriate Medication Use Scale, ${ }^{39}$ distress measured by the HADS, ${ }^{26}$ beliefs about AET measured by the Beliefs about Medicines Questionnaire-AET, ${ }^{40}$ social support measured by the Multidimensional Scale of Perceived Social Support, ${ }^{41}$ acquired coping skills measured by the Measure of Current Status-part A, ${ }^{42}$ selfefficacy for symptom management measured by the SelfEfficacy For Managing Symptoms Questionnaire, ${ }^{43}$ and cognitive functioning measured by the Patient-Reported Outcomes Measurement Information System-Cognitive Function-Short Form 4a. ${ }^{44}$

\section{Safety and adverse events}

Study staff will review self-report measures on completion for missing data and to monitor distress levels. If a patient endorses a score $\geq 11$ on the depression subscale of the HADS, the PI will be notified and either the PI or one of the trained study therapists will contact the patient via telephone to conduct a risk/safety assessment. If the patient requires further outpatient services (eg, pharmacotherapy) or is at risk for self-harm, study staff will make the necessary referrals for treatment and/or urgent psychiatric care. If suicidality or risk of harm to others is otherwise discovered at any study visit, the patient will be referred to the appropriate services.

At weekly meetings, the research team will discuss summaries of adverse events by treatment group, and all serious adverse events will be reported to the PI, the DF/HCC Institutional Review Board (IRB) and the appropriate federal agencies (eg, National Cancer Institute) regardless of any judgement of their relatedness to the study. The research team will also discuss summary reports of treatment retention and reasons for dropout or withdrawal by treatment group. Patients who withdraw will be asked if they are willing to complete assessment measures. The project will be stopped immediately if at any point the DF/HCC IRB or the study investigators judge that the risks of study procedures outweigh the benefits. Furthermore, the DF/HCC IRB will conduct trial auditing if deemed necessary throughout the study.

\section{Data collection and management}

The study PI will oversee all aspects of data collection and management. All data management activities will use REDCap for electronic collection and management of 
data. Data management reports will be generated weekly and discussed during the study team meetings. Study source documents, including but not limited to signed consent forms, completed eligibility checklists and any paper-based self-report questionnaires, will be scanned and stored digitally as certified copies on a secure drive within the encrypted MGH network accessible only to trained study staff. Physical source documents will be destroyed once they are scanned and the corresponding electronic document is confirmed to be viable. If a patient is consented electronically, their digital consent form will be saved as the original source document.

\section{Statistical analysis}

The primary objective of this pilot study is to demonstrate feasibility, defined as (1) enrolment rate $>50 \%$, (2) retention rate $>70 \%$ and (3) intervention attendance rate $\geq 70 \%$ (e, $\geq 70 \%$ of participants complete at least four of six sessions).

For the analysis of secondary outcomes of the STRIDE intervention on objective and subjective adherence to AET, symptom-related distress and satisfaction with AET, data will be first be assessed for patterns of missingness ${ }^{45}$ and statistical assumptions. We will use the intention-totreat principle for analyses with all randomised subjects and maximum likelihood with multiple imputation to account for missing data. We will then conduct mixedeffects models with repeated measures data for secondary outcomes and relevant demographic and treatmentrelated factors as covariates (eg, age, stage, time since AET initiation). Longitudinal analyses will include all time points and a cross-sectional analysis for each time point. Given that this pilot is not powered to detect statistically significant group differences, we will examine mean differences and sample variability on the secondary outcomes. Effect sizes (Cohen's d) will be calculated for changes in outcomes from baseline to 12 and 24 weeks, where 0.3 indicates a small effect, 0.5 a medium effect and 0.8 a large effect. ${ }^{46}$ Effect sizes will be used in a power analysis to estimate the necessary sample size to conduct a full-scale efficacy trial with power $>80 \%$. We will also verify the needed sample size based on ability to detect a clinically meaningful difference and references to existing literature. ${ }^{47}$ We will use the same statistical procedures to examine group differences on exploratory outcomes (eg, QOL, distress). Finally, we will explore (1) possible mediators of the intervention (eg, coping ability) by examining the bootstrapped confidence intervals of the indirect effects and (2) possible moderators (eg, social support) of intervention effects by probing interactions between the moderator and group assignment that reach or approach significance $(\mathrm{p}<0.15)$ when predicting patient-reported outcomes.

\section{Limitations}

Limitations of the current trial deserve mention. First, the study is limited to patients with breast cancer currently receiving care at a Boston area major medical centre and its affiliate sites, limiting the generalisability of the results. Second, patients who have access to devices (eg, smartphones, computers, tablets) with videoconferencing capability are more likely to participate, which may bias the sample to individuals with higher incomes and education. However, videoconference delivery does have the potential to increase accessibility for individuals for whom in-person sessions may be prohibitive due to travel and/or associated costs. Third, although we are using a randomised, controlled design, both the participants and the study therapists are aware of each patient's study group assignment after randomisation, as it is not possible to conduct a blinded psychosocial intervention study. Fourth, while we considered an attention-matched condition to control for nonspecific intervention effects, we decided against this approach because medication monitoring is likely to improve adherence alone. ${ }^{30}$ Additionally, requesting participation in a placebo intervention adds undue burden to patients who are coping with emotional and physical sequelae. In fact, an attention control should only be employed if attention (from the therapist) would affect the primary outcome. Since no data suggest that therapist attention would improve AET adherence, an attention control would be an added expense and potentially unethical. ${ }^{30}$ While many patients continue to take AET for up to 10 years, our assessment follow-ups are limited to approximately 6 months following enrolment. Future studies may benefit from a longer follow-up period. Finally, although the study is underpowered to examine group differences in secondary outcomes, the purpose of including all study questionnaires is to also examine the feasibility of participants completing an assessment battery of this size. If participants do not complete the surveys, this may indicate an unacceptable lengthiness that would prohibit data collection and analysis in a future full-scale efficacy trial. Survey length was directly assessed in the run-in phase of this study.

\section{Ethics and dissemination}

The trial is being conducted in compliance with this study protocol, which has been approved by the DF/HCC's IRB. Written informed consent is and will be obtained from every participant, either in-person or over the phone; if consented over the phone, participants are and will be provided with a secure electronic consent form that is digitally signed. All documents, investigative reports, and information relating to the participants are confidential. Participant data are deidentified and compliant with the Standards of Privacy of Individually Identifiable Health Information ('Privacy Rule') of Health Insurance Portability and Accountability Act. Significant modifications to the study protocol will be submitted to the DF/HCC IRB for approval and communicated to study participants and all relevant members of the research team. Due to this being a low-risk, social/behavioural intervention, it is unlikely participants will be at any risk of physical harm because of study participation but they may experience 
increased emotional distress as a result of the intervention content. Participants may withdraw from the study at any time, and participants for whom distress is not adequately resolved will be offered additional psychological care through the primary site's cancer centre. Any results from this study will be published in peer-reviewed journals and local and national conference proceedings, and a description of the trial and summary of results will be available on ClinicalTrials.gov. The investigative team will track the manuscript in accordance with authorship guidelines. Only the investigative team will have access to the trial dataset, and access will only be permitted on reasonable request through a data usage agreement via the IRB. There are no plans for the use of professional writers. If a patient expresses interest in learning the results of the study, study staff will provide an abstract of the study results on completion of data collection.

\section{Current trial status}

Recruitment of participants started on 11 October 2019, and as of 15 March 2020, 25 patients had enrolled. The trial was put on a temporary recruitment pause on 26 March 2020 due to the COVID-19 pandemic and plans to resume recruitment in June 2020.

\section{Author affiliations}

${ }^{1}$ Department of Psychiatry, Massachusetts General Hospital, Boston, Massachusetts, USA

${ }^{2}$ Department of Psychiatry, Harvard Medical School, Boston, Massachusetts, USA ${ }^{3}$ Department of Psychology, Temple University, Philadelphia, Pennsylvania, USA ${ }^{4}$ Department of Psychology, University of Miami, Coral Gables, Florida, USA ${ }^{5}$ Medicine, Harvard Medical School, Boston, Massachusetts, USA

${ }^{6}$ Medicine, Massachusetts General Hospital Cancer Center, Boston, Massachusetts, USA

Correction notice This article has been corrected since it first published. The provenance and peer review statement has been included.

Acknowledgements We thank the study patients for their time and dedication to this research study.

Contributors JJ, CR, AH, MC, EW, JP, JT and JG contributed to the study conception and design. The protocol was developed by JJ, CR, AH, MC, EW, JP, JT and $\mathrm{JG}$, and written by JJ, EW and CR. The first draft of the manuscript was written by JJ, CR, AH and JJ, CR, AH, MC, EW, JP, JT and JG commented on previous versions of the manuscript. JJ, CR, AH, MC, EW, JP, JT and JG read and approved the final manuscript.

Funding This work was supported by a Career Development Award from the National Cancer Institute of the National Institutes of Health (K07CA211107; Jacobs).

Competing interests $\mathrm{JJ}$ is a paid consultant from Blue Note Therapeutics. JG receives royalties from Springer Humana Press, has research funding from Gaido Health/BCG Digital Ventures, and is a paid consultant from Concerto HealthAl and Blue Note Therapeutics. JP has received research funding from Pfizer, is a paid consultant for Athenex, and JP's spouse is an employee of GlaxoSmithKline. All other authors declare that they have no conflicts of interest.

Patient and public involvement Patients and/or the public were involved in the design, or conduct, or reporting, or dissemination plans of this research. Refer to the Methods section for further details.

Patient consent for publication Not required.

Provenance and peer review Not commissioned; externally peer reviewed.

Open access This is an open access article distributed in accordance with the Creative Commons Attribution Non Commercial (CC BY-NC 4.0) license, which permits others to distribute, remix, adapt, build upon this work non-commercially, and license their derivative works on different terms, provided the original work is properly cited, appropriate credit is given, any changes made indicated, and the use is non-commercial. See: http://creativecommons.org/licenses/by-nc/4.0/.

ORCID iD

Jamie M Jacobs http://orcid.org/0000-0001-9740-624X

\section{REFERENCES}

1 Burstein HJ, Temin S, Anderson H, et al. Adjuvant endocrine therapy for women with hormone receptor-positive breast cancer: American Society of clinical oncology clinical practice guideline focused update. J Clin Oncol 2014;32:2255-69.

2 , Davies C, Godwin J, et al, Early Breast Cancer Trialists' Collaborative Group (EBCTCG). Relevance of breast cancer hormone receptors and other factors to the efficacy of adjuvant tamoxifen: patient-level meta-analysis of randomised trials. Lancet 2011;378:771-84.

3 Early Breast Cancer Trialists' Collaborative Group (EBCTCG). Aromatase inhibitors versus tamoxifen in early breast cancer: patient-level meta-analysis of the randomised trials. Lancet 2015;386:1341-52.

4 Chlebowski RT, Kim J, Haque R. Adherence to endocrine therapy in breast cancer adjuvant and prevention settings. Cancer Prev Res 2014;7:378-87.

5 Markkula A, Hietala M, Henningson M, et al. Clinical profiles predict early nonadherence to adjuvant endocrine treatment in a prospective breast cancer cohort. Cancer Prev Res 2012;5:735-45.

6 Markkula A, Hietala M, Henningson M, et al. Clinical profiles predict early nonadherence to adjuvant endocrine treatment in a prospective breast cancer cohort. Cancer Prev Res 2012;5:735-45.

7 Yood MU, Owusu C, Buist DSM, et al. Mortality impact of less-thanstandard therapy in older breast cancer patients. J Am Coll Surg 2008;206:66-75.

8 DiMatteo MR, Giordani PJ, Lepper HS, et al. Patient adherence and medical treatment outcomes: a meta-analysis. Med Care 2002;40:794-811.

9 Waterhouse DM, Calzone KA, Mele C, et al. Adherence to oral tamoxifen: a comparison of patient self-report, pill counts, and microelectronic monitoring. J Clin Oncol 1993;11:1189-97.

10 Makubate B, Donnan PT, Dewar JA, et al. Cohort study of adherence to adjuvant endocrine therapy, breast cancer recurrence and mortality. Br J Cancer 2013;108:1515-24.

11 Hadji P, Blettner M, Harbeck N, et al. The patient's anastrozole compliance to therapy (PACT) program: a randomized, in-practice study on the impact of a standardized information program on persistence and compliance to adjuvant endocrine therapy in postmenopausal women with early breast cancer. Ann Oncol 2013;24:1505-12.

12 Czajkowski SM, Powell LH, Adler N, et al. From ideas to efficacy: the orbit model for developing behavioral treatments for chronic diseases. Health Psychol 2015;34:971-82.

13 Yu K-D, Zhou Y, Liu G-Y, et al. A prospective, multicenter, controlled, observational study to evaluate the efficacy of a patient support program in improving patients' persistence to adjuvant aromatase inhibitor medication for postmenopausal, early stage breast cancer. Breast Cancer Res Treat 2012;134:307-13.

14 Davies C, Pan H, Godwin J, et al. Long-Term effects of continuing adjuvant tamoxifen to 10 years versus stopping at 5 years after diagnosis of oestrogen receptor-positive breast cancer: atlas, a randomised trial. Lancet 2013;381:805-16.

15 Stanton AL, Petrie KJ, Partridge AH. Contributors to nonadherence and nonpersistence with endocrine therapy in breast cancer survivors recruited from an online research registry. Breast Cancer Res Treat 2014;145:525-34.

16 Moon Z, Moss-Morris R, Hunter MS, et al. Barriers and facilitators of adjuvant hormone therapy adherence and persistence in women with breast cancer: a systematic review. Patient Prefer Adherence 2017;11:305-22.

17 Neven P, Markopoulos C, Tanner M, et al. The impact of educational materials on compliance and persistence rates with adjuvant aromatase inhibitor treatment: first-year results from the compliance of aromatase inhibitors assessment in daily practice through educational approach (CARIATIDE) study. Breast 2014;23:393-9.

18 Greer JA, Amoyal N, Nisotel L, et al. A systematic review of adherence to oral antineoplastic therapies. Oncologist 2016;21:354-76.

19 Onken LS, Carroll KM, Shoham V, et al. Reenvisioning clinical science: unifying the discipline to improve the public health. Clin Psychol Sci 2014;2:22-34. 
20 Rounsaville BJ, Carroll KM, Onken LS. A stage model of behavioral therapies research: getting started and moving on from stage I. Clin Psychol: Sci Pract 2001;8:133-42.

21 Jacobs JM, Walsh EA, Park ER, et al. The patient's voice: adherence, symptoms, and distress related to adjuvant endocrine therapy after breast cancer. Int J Behav Med 2020;27:687-97.

22 Antoni MH, Smith R. Stress Management Intervention for Women with Breast Cancer: Participant's Workbook. Washington, DC: American Psychological Association, 2003.

23 Safren SA, Hendriksen ES, Mayer $\mathrm{KH}$, et al. Cognitive-Behavioral therapy for HIV medication adherence and depression. Cogn Behav Pract 2004;11:415-24.

24 Dabrowski M, Boucher K, Ward JH, et al. Clinical experience with the NCCN distress thermometer in breast cancer patients. J Natl Compr Canc Netw 2007;5:104-11.

25 Farmer KC. Methods for measuring and monitoring medication regimen adherence in clinical trials and clinical practice. Clin Ther 1999;21:1074-90.

26 Zigmond AS, Snaith RP. The hospital anxiety and depression scale. Acta Psychiatr Scand 1983;67:361-70.

27 Harris PA, Taylor R, Thielke R, et al. Research electronic data capture (REDCap)--a metadata-driven methodology and workflow process for providing translational research informatics support. J Biomed Inform 2009;42:377-81.

28 Safren SA, Soroudi N, Gonzalez JS. Coping with chronic illness : a cognitive-behavioral therapy approach for adherence and depression workbook. New York: Oxford University Press, 2007.

29 Wickersham K, Colbert A, Caruthers D, et al. Assessing fidelity to an intervention in a randomized controlled trial to improve medication adherence. Nurs Res 2011;60:264-9.

30 Pagoto SL, McDermott MM, Reed G, et al. Can attention control conditions have detrimental effects on behavioral medicine randomized trials? Psychosom Med 2013;75:137-43.

31 Attkisson CC, Zwick R. The client satisfaction questionnaire. psychometric properties and correlations with service utilization and psychotherapy outcome. Eval Program Plann 1982;5:233-7.

32 DiMatteo MR, Lepper HS, Croghan TW. Depression is a risk factor for noncompliance with medical treatment: meta-analysis of the effects of anxiety and depression on patient adherence. Arch Intern Med 2000;160:2101-7.

33 Thivat E, Van Praagh I, Belliere A, et al. Adherence with oral oncologic treatment in cancer patients: interest of an adherence score of all dosing errors. Oncology 2013;84:67-74.

34 Partridge $\mathrm{AH}$, Archer L, Kornblith AB, et al. Adherence and persistence with oral adjuvant chemotherapy in older women with early-stage breast cancer in CALGB 49907: adherence companion study 60104. J Clin Oncol 2010;28:2418-22.

35 Horne R, Weinman J. Self-Regulation and self-management in asthma: exploring the role of illness perceptions and treatment beliefs in explaining non-adherence to preventer medication. Psychol Health 2002;17:17-32.

36 Cella D, Land SR, Chang C-H, et al. Symptom measurement in the breast cancer prevention trial (BCPT) (P-1): psychometric properties of a new measure of symptoms for midlife women. Breast Cancer Res Treat 2008;109:515-26.

37 Abetz L, Coombs JH, Keininger DL, et al. Development of the cancer therapy satisfaction questionnaire: item generation and content validity testing. Value Health 2005;8 Suppl 1:S41-53.

38 Brady MJ, Cella DF, Mo F, et al. Reliability and validity of the functional assessment of cancer Therapy-Breast quality-of-life instrument. J Clin Oncol 1997;15:974-86.

39 Risser J, Jacobson TA, Kripalani S. Development and psychometric evaluation of the self-efficacy for appropriate medication use scale (SEAMS) in low-literacy patients with chronic disease. J Nurs Meas 2007;15:203-19.

40 Brett J, Hulbert-Williams NJ, Fenlon D, et al. Psychometric properties of the beliefs about medicine Questionnaireadjuvant endocrine therapy (BMQ-AET) for women taking AETs following early-stage breast cancer. Health Psychol Open 2017;4:205510291774046.

41 Zimet GD, Dahlem NW, Zimet SG, et al. The multidimensional scale of perceived social support. J Pers Assess 1988;52:30-41.

42 Antoni MH, Lechner SC, Kazi A, et al. How stress management improves quality of life after treatment for breast cancer. J Consult Clin Psychol 2006;74:1143-52.

43 Shelby RA, Edmond SN, Wren AA, et al. Self-Efficacy for coping with symptoms moderates the relationship between physical symptoms and well-being in breast cancer survivors taking adjuvant endocrine therapy. Support Care Cancer 2014;22:2851-9.

44 Lai J-S, Wagner LI, Jacobsen PB, et al. Self-Reported cognitive concerns and abilities: two sides of one coin? Psychooncology 2014;23:1133-41.

45 Little RJ, D'Agostino R, Cohen ML, et al. The prevention and treatment of missing data in clinical trials. $N$ Engl $\mathrm{J}$ Med 2012;367:1355-60.

46 Cohen J. A power primer. Psychol Bull 1992;112:155-9.

47 Kraemer HC, Mintz J, Noda A, et al. Caution regarding the use of pilot studies to guide power calculations for study proposals. Arch Gen Psychiatry 2006;63:484-9. 